\title{
OPEN Use of open source monitoring hardware to improve the production of MOFs: using STA-16(Ni) as a case study
}

Felicity Massingberd-Mundy $y^{1,2}$, Stephen Poulston ${ }^{1}$, Stephen Bennett ${ }^{1}$, Hamish Hei-Man Yeung ${ }^{2,3}$ \& Timothy Johnson ${ }^{1}{ }^{1 凶}$

Affordable and readily available microelectronics are becoming prevalent in teaching laboratories however these useful and economic tools are not used widely in either academia or industry. Herein we report how a metal organic framework (MOF) synthetic route can be optimized using an in situ monitoring apparatus designed in-house on open source hardware for under $\$ 100$. We demonstrate that the MOF can be produced at atmospheric pressure, an improvement over previous reports, but also with a reduction in reaction time of $93 \%$. This improvement in reaction time was predicted after a single experiment using the monitoring kit showing how efficiencies in the lab can be gained with very little experimental and monetary overhead while minimising the resources used.

The increasing availability of affordable open source microelectronics presents research scientists with a unique opportunity to prototype instrumentation specifically designed around their research needs. Bespoke apparatus offers many advantages over commercial offerings-with flexibility, cost and ownership being key. A growing number of reports have demonstrated the use of these types of apparatus in education ${ }^{1,2}$, notably the ability to produce systems for students with disabilities ${ }^{3,4}$. Several reports have also demonstrated how such equipment can be used outside the teaching laboratories with the development of potentiometric detection ${ }^{5}$, scanning electrochemical microscopes ${ }^{6}$ and electrochemical pre-treatment apparatus ${ }^{7}$ all using inexpensive and open source hardware solutions.

One area of interest to materials research scientists is the ability to log data from a range of sensors to gain insight into a reaction mixture, in situ. Mercer et al. demonstrated that a cost-effective device could be produced using Internet of Things (IoT) connected microcontrollers that could log turbidity data ${ }^{8}$. While insight gained into the precipitation of $\mathrm{NaCl}$ from $\mathrm{Na}_{2} \mathrm{~S}_{2} \mathrm{O}_{3}$ and $\mathrm{HCl}$ was useful in the context of chemical education, insight gained from this type of measurement may also be of interest to scientists studying novel systems.

Within our laboratory the production and optimization of metal organic frameworks is an important and active area $^{9}$. MOFs are a possible disruptive innovation with applications including gas separation, catalysis, drug delivery and medical devices ${ }^{10-13}$. These materials are becoming a target for commercialization as their applications are continually demonstrated. While much work has focused on design ${ }^{14}$ and fundamental understanding ${ }^{15}$, commercialization of these preparation routes is not a trivial task. Poor solvent choice, low concentrations and the use of pressure vessels plague the literature. Optimization of preparation routes is key if a given material is to be of commercial relevance.

Time-resolved ex situ and in situ techniques have been used to investigate MOF crystallization mechanisms and reaction intermediates-key aspects of material scale-up ${ }^{16}$. Such techniques include X-ray diffraction, smallangle and wide-angle X-ray scattering (SAXS and WAXS), NMR, dynamic and static light scattering (DLS and SLS), and scanning and transmission electron microscopy (SEM and TEM). The downside to these techniques is that they are often expensive and complicated to use, requiring specific training.

Recently, Yeung et al. used in situ synchrotron XRD to monitor the formation of ZIF-8, shedding light on its complex crystallization mechanism ${ }^{17}$. In situ laboratory $\mathrm{pH}$ measurements and turbidity measurements, the

\footnotetext{
${ }^{1}$ Johnson Matthey Technology Centre, Blount's Court, Sonning Common, Reading RG4 9NH, UK. ${ }^{2}$ Inorganic Chemistry Laboratory, University of Oxford, South Parks Road, Oxford OX1 30R, UK. ${ }^{3}$ Present address: School of Chemistry, University of Birmingham, Edgbaston, Birmingham B15 2TT, UK. 『email: timothy.johnson@ matthey.com
} 
<smiles>O=P(O)(O)CN1CCC(C2CCN(CP(=O)(O)O)CC2)CC1</smiles>

a)

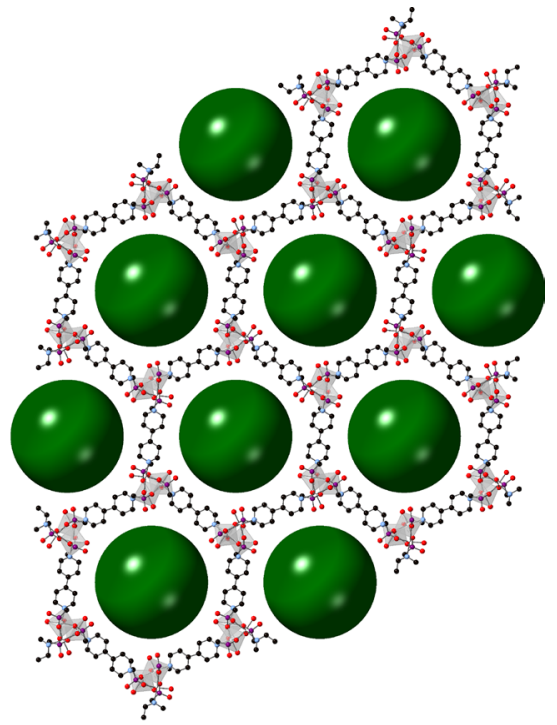

b)

Figure 1. (a) STA-16 linker and (b) representation of the STA-16(Ni) framework. Black, light blue, red and purple spheres represent carbon, nitrogen, oxygen and phosphorous atoms respectively. Ni-O polyhedra are represented in light grey and green spheres $(\mathrm{r}=2 \mathrm{~nm})$ are used to illustrate the pore voids. Protons omitted for clarity.

latter performed using a low-cost Raspberry Pi-based apparatus, showed similar trends to XRD, suggesting that important insights could be gained from the use of cheaper consumer electronics alone.

To understand how in situ reaction monitoring could be used to specifically optimize MOF production routes, the STA-16(Ni) system was studied. This material consists of octahedra metal centers connected to the linker $N, N^{\prime}-4,4^{\prime}$-bipiperidinebis(methylenephosphonic acid), Fig. 1a, further referred to as STA-16 Linker.

STA-16 is an exceptional material due to its large pore size and diverse range of metal centers-with Co, $\mathrm{Ni}$, $\mathrm{Fe}, \mathrm{Mn}$, and $\mathrm{Mg}$ reported ${ }^{18,19}$. A representation of the framework can be seen in Fig. 1b. The dehydrated forms of these materials have coordinatively unsaturated $\operatorname{sites}^{18,20,21}$, with the consequential Lewis acidity resulting in catalytic applications for these structures ${ }^{19,22}$. These MOFs have also been investigated for use in gas adsorption and separation ${ }^{18,21,23}$. The larger pores of the STA-16 structure make it suited to capturing larger molecules ${ }^{20}$. This makes the STA-16 frameworks particularly interesting for industrial exploration due to its potential use in a diverse range of applications. While this framework is of interest, its use is stymied by its production using pressure vessels and a reported reaction time of $72 \mathrm{~h}$-limiting both space and time yields ${ }^{20}$. Ultimately, improving the reaction procedure for this material will signify a significant cost saving as well as a reduction in energy and resource usage. We report the design of a multi-parameter in situ monitoring apparatus constructed from open source hardware and its use in the optimization of the synthesis of STA-16(Ni). We find that the material can be produced with a reduction in reaction time of $93 \%$ over the reported procedure, without the use of pressure vessels. We believe this is the first example of an instrument (which is bespoke, produced on a budget and open source) being used to investigate and improve the production of a material of industrial relevance resulting in the delivery of quality material in a speedy and economic fashion.

\section{Methods}

Materials. Hydrobromic acid solution, phosphorous acid, 4,4'-bipiperidine dihydrochloride and nickel(II) acetate tetrahydrate were purchased from Alfa Aesar with formaldehyde and KOH obtained from Fisher Scientific. All chemicals were used without further purification.

STA-16 linker. The STA-16 linker was produced using a modified version from past reports ${ }^{20}$. In brief, 4,4'-bipiperidine dihydrochloride (14.35 g, $0.06 \mathrm{~mol})$ and phosphorous acid (12.8 g, $0.156 \mathrm{~mol})$ were dissolved in distilled water $(60 \mathrm{ml})$. To this hydrobromic acid solution $(42 \mathrm{ml} ; 48 \mathrm{wt} \%$ aqueous solution) was added and the mixture was stirred until all the solids had dissolved. Formaldehyde ( $26 \mathrm{ml}, 0.35 \mathrm{~mol} ; 35 \mathrm{wt} \%$ aqueous solution) was added dropwise to the reaction mixture over $30 \mathrm{~min}$ and the solution was heated to $120^{\circ} \mathrm{C}$ for $20 \mathrm{~h}$. The reaction mixture was chilled at $-22^{\circ} \mathrm{C}$ overnight to facilitate full crystallization. The product was separated by vacuum filtration and washed with cold ethanol:water mixtures $(90: 10,3 \times 30 \mathrm{ml})$ prior to drying at $40{ }^{\circ} \mathrm{C}$ overnight in static air.

STA-16(Ni) synthesis. STA-16(Ni) was produced at various scales with exact quantities for each reaction shown in Table 1. An example synthesis for Entry 1 is as follows. STA-16 Linker $(5.18 \mathrm{~g}, 0.42 \mathrm{mmol})$ was added to $\mathrm{H}_{2} \mathrm{O}(250 \mathrm{ml})$. To this $\mathrm{KOH}(29.75 \mathrm{ml}, 1.0 \mathrm{M})$ was added followed by nickel(II) acetate tetrahydrate 


\begin{tabular}{|l|c|l|l|l|}
\hline Entry & Linker $(\mathbf{g})$ & $\mathbf{H}_{\mathbf{2}} \mathbf{O}(\mathbf{m l})$ & $\mathbf{1} \mathbf{M ~ K O H ~}(\mathbf{m l})$ & Ni acetate $(\mathbf{g})$ \\
\hline 1 & 5.18 & 250 & 29.75 & 7 \\
\hline 2 & 10.36 & 500 & 60 & 14 \\
\hline 3 & 0.52 & 25 & 3 & 0.7 \\
\hline
\end{tabular}

Table 1. Reagent quantities to produce STA-16(Ni).

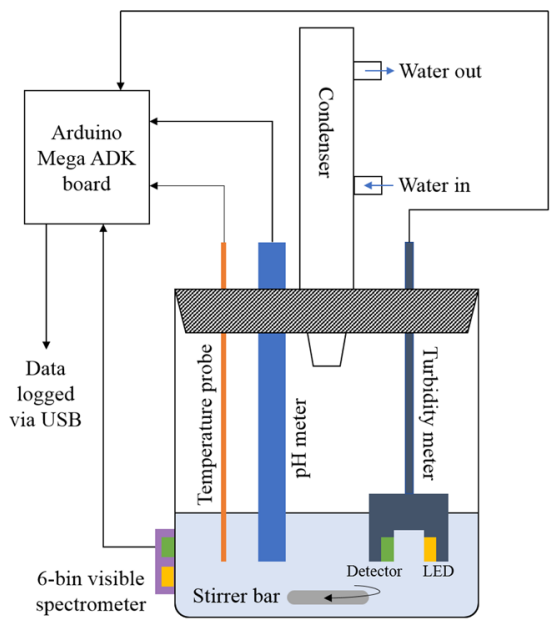

a)

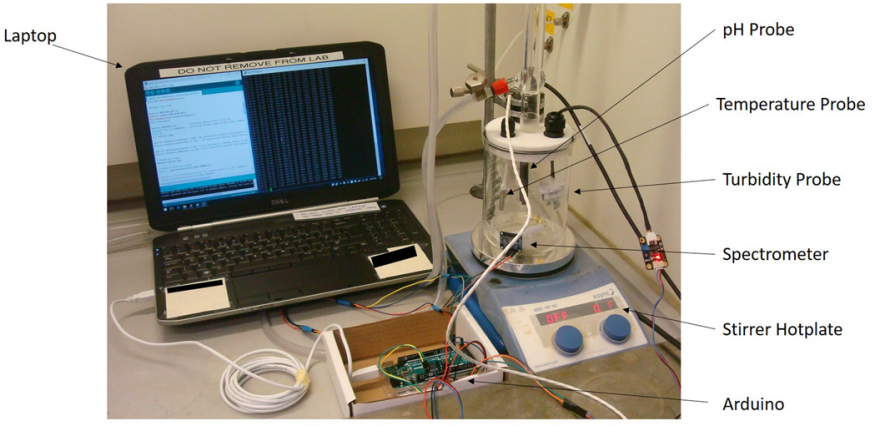

b)

Figure 2. (a) Schematic representation and (b) a photograph of the Arduino ${ }^{\circledast}$ in situ monitoring apparatus.

(7 g, $0.82 \mathrm{mmol}$ ). The reaction mixture was aged at room temperature for $45 \mathrm{~min}$ before heating to $120^{\circ} \mathrm{C}$ for $65.25 \mathrm{~h}-\mathrm{a}$ total reaction time of $66 \mathrm{~h}$. Once cooled the sample was separated by filtration and washed three times with $1 \mathrm{M} \mathrm{KOH}$ followed by two washings with water. The material was activated under vacuum at $120^{\circ} \mathrm{C}$ overnight prior to further analysis.

ZIF-8 synthesis. The production of ZIF-8 was modified from previous reports ${ }^{24}$. In brief-solutions of $\mathrm{Zn}\left(\mathrm{NO}_{3}\right)_{2} \cdot 6 \mathrm{H}_{2} \mathrm{O}(1.40 \mathrm{~g}, 4.7 \mathrm{mmol})$ in methanol $(95 \mathrm{ml})$ and 2-methylimidazole $(3.09 \mathrm{~g}, 37.6 \mathrm{mmol})$ in methanol $(95 \mathrm{~mL})$ were prepared. The in situ $\mathrm{pH}$, turbidity and temperature probes were then placed in the 2-methylimidazole solution. Once the $\mathrm{pH}$ and turbidity had stabilized, the zinc nitrate solution was added under stirring. The resulting product was collected by centrifugation and the sediment was washed 3 times with methanol. The product was dried in air at room temperature overnight. The sample was activated at $50{ }^{\circ} \mathrm{C}$ overnight prior to characterization.

Arduino ${ }^{\circledR}$ apparatus. Figure 2a shows a schematic representation of the in situ monitoring apparatus with Fig. $2 \mathrm{~b}$ showing a photograph of the apparatus. The apparatus consists of an Arduino ${ }^{\circ}$ Mega prototyping board with $\mathrm{pH}$, turbidity and temperature sensors. These sensors are placed within the reaction media. A six-channel visible light spectrometer was also connected and secured to the outside of the reaction vessel. Color readings of the reaction mixture, along with temperature, turbidity and $\mathrm{pH}$ are all recorded by the Arduino ${ }^{\circ}$ and stored on the attached PC.

Before each experiment the turbidity sensor was calibrated using Formazin turbidity standards of 1, 5, 10, 100, 1000, 2000, and 4000 Nephelometric Turbidity Units (NTUs), and the $\mathrm{pH}$ meter was calibrated against buffer solutions of $\mathrm{pH} 4.02,7.00,9.00$, and 10.00. Calibration curves were produced by plotting the output of the turbidity meter against the turbidity of the standard, and the output of the $\mathrm{pH}$ meter against the $\mathrm{pH}$ of the buffer solution. An exponential fitting was used for the turbidity calibration curve. A linear fitting was used for the $\mathrm{pH}$ meter calibration as this produced the highest $R^{2}$ value.

The temperature probe was calibrated against digital thermometer readings of a heated water sample and the readings of the probe were found to be accurate to $\pm 0.5^{\circ} \mathrm{C}$. The derived parameters from the turbidity calibration and $\mathrm{pH}$ calibration fittings were applied within the code for the Arduino ${ }^{\circ}$ board to convert the analogue $\mathrm{pH}$ and turbidity outputs into readings in units of $\mathrm{pH}$ and NTUs respectively.

In situ visible spectroscopy measurements were recorded during the MOF syntheses. The spectrometer was secured to the outside of the glass reaction vessel. Luminance was measured in units of $\mu \mathrm{W} \mathrm{cm}{ }^{-2}$. A time-resolved spectrum was then produced by plotting the normalised luminance against time. 


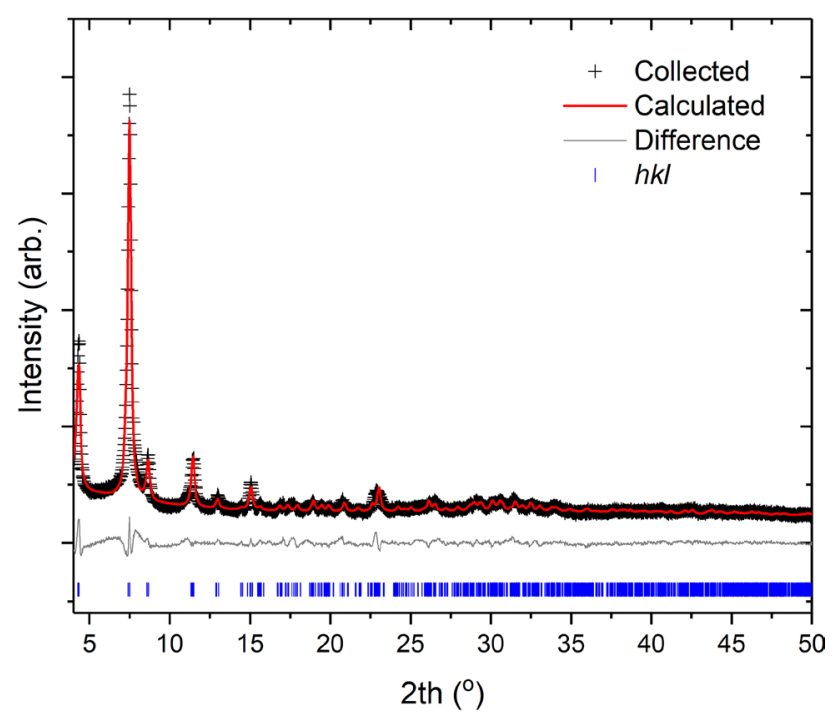

Figure 3. Collected PXRD pattern and subsequent Rietveld refinement for STA-16(Ni) produced at $62 \mathrm{~h}$ under reflux conditions (Entry 1).

XRD. Powder X-ray diffraction (PXRD) data were collected in reflection geometry using a Bruker AXS D8 diffractometer using $\mathrm{Cu} \mathrm{Ka}$ radiation $(\lambda=1.5406+1.54439 \AA)$ over the $5<2 \theta<50^{\circ}$ range in $0.02^{\circ}$ steps. Refinement was performed using Topas ${ }^{25}$ with reflection profiles modelled using a fundamental parameters approach ${ }^{26}$ with reference data collected from NIST660 $\mathrm{LaB}_{6}$.

Physisorption. Samples were subjected to analysis by $\mathrm{N}_{2}$ physisorption. Isotherms were collected on a TriStar II instrument with experiments being conducted at $-196^{\circ} \mathrm{C}$ in $\mathrm{N}_{2}$. All samples were degassed at $120^{\circ} \mathrm{C}$ for $12 \mathrm{~h}$ prior to analysis.

\section{Results and discussion}

Previous reports of the STA-16(Ni) framework rely on the use of autogenous pressure to produce the framework. Initial work conducted for this study focused on producing the material using conditions that are more amenable to large scale production. To this end the preparation route was successfully conducted at atmospheric pressure under reflux conditions. Table 1 Entry 1 shows the quantities of reagents used for this reaction. The STA-16 linker required for these experiments was produced in accordance to literature reports ${ }^{20}$. The collected PXRD and subsequent Rietveld refinement can be seen in Supplementary Fig. S1 online. This shows a phase pure linker was obtained.

Figure 3 shows the collected PXRD and subsequent Rietveld refinement demonstrating a phase pure STA$16(\mathrm{Ni})$ material has been produced. While this route is not optimized, with a yield of only $18.35 \%$, it does demonstrate this material can be produced in an agreeable manner without the need for expensive pressure vessels with limited space time yields.

While demonstrating the material can be produced at atmospheric pressure under reflux conditions, a significant improvement over literature reports, the framework still required $66 \mathrm{~h}$ to produce. This is not acceptable if the material is to be produced commercially. For this reason, the preparation route was subjected to in situ reaction monitoring.

The bespoke reaction monitoring apparatus, shown in Fig. 2, was used to monitor the formation of the STA$16(\mathrm{Ni})$ framework. The key to this piece of novel apparatus is the ability to connect, log and analyze data from a range of different commercially available sensors. The apparatus can simultaneously monitor $\mathrm{pH}$, turbidity, temperature and the visible spectrum of a reaction mixture. This approach means that, even when very little physical information is known about the system, the chance of observing a reaction critical process is high.

The code used to collect the in situ data is reproduced in Supplementary Fig. S2 online. The apparatus was produced and coded for under \$100-with a parts list shown in Table 2. This makes it ideal for monitoring reactions of interest in the lab regardless of budget.

In situ turbidity data, Fig. 4, show clearly how, during the initial $45 \mathrm{~min}$ of aging, the reaction mixture becomes more turbid. Upon heating the reaction mixture reaches a maximum value of $\sim 9000$ NTU. After this a drop in turbidity is noted before stabilizing. This drop is likely due to a critical concentration of a metal-linker species producing large solid particles of MOF which reduce the turbidly of the overall solution. Additionally, $\mathrm{pH}$ and spectrometer data show similar responses. Supplementary Fig. S3 online shows data collected from the $\mathrm{pH}$ and spectrometer sensors during the reaction. The $\mathrm{pH}$ data is consistent with the formation of the MOF and the liberation of protons from the used linker- this effect reaches equilibrium after $5 \mathrm{~h}$. Additionally, the spectrometer data show how changes can be observed in the sensor response during the aging and heating segments of the reaction. This is in line with empirical observations as the reaction mixture goes from colorless to green on 


\begin{tabular}{|l|l|l|l|}
\hline Part & Manufacturer & Product code & Price (USD) \\
\hline Turbidity probe & DFRobot & SEN0189 & 10 \\
\hline Thermocouple & Adafruit & DS18B20 & 7 \\
\hline 6-Channel visible light sensor & Adafruit & AS7262 & 20 \\
\hline pH Probe & DFRobot & SEN0161 & 30 \\
\hline Arduino ${ }^{\circ}$ mega & Arduino $^{\circ}$ & A000067 & 31 \\
\hline
\end{tabular}

Table 2. Parts list for the in situ monitoring device. Pricing from manufacture at time of writing.

(a)

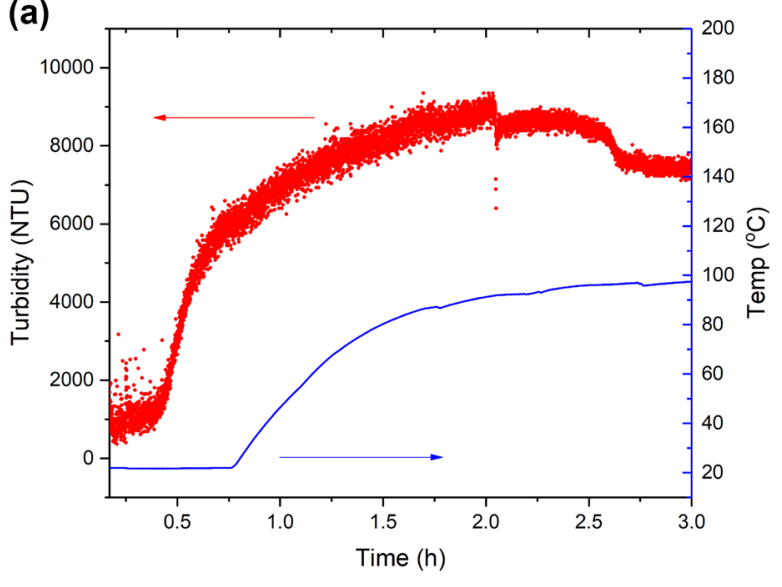

(b)

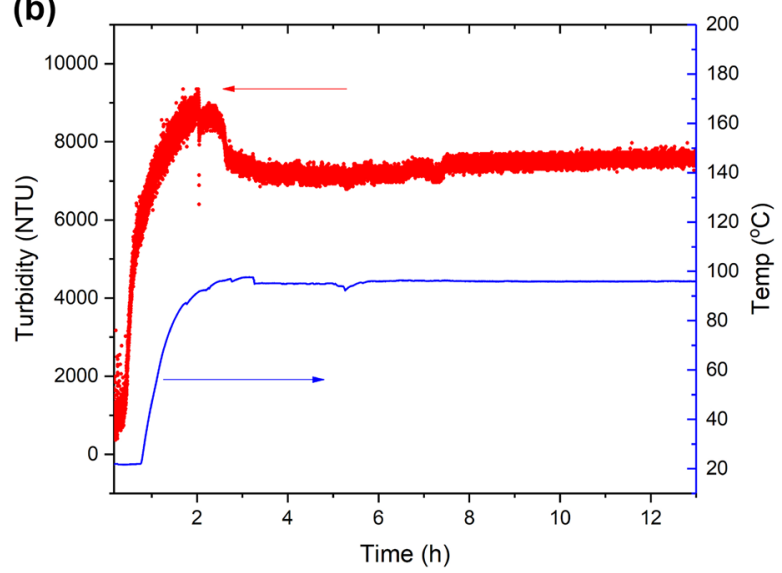

Figure 4. (a) Turbidity and temperature data obtained from the in situ reaction monitoring kit, and (b) the same data with an extended time axis.

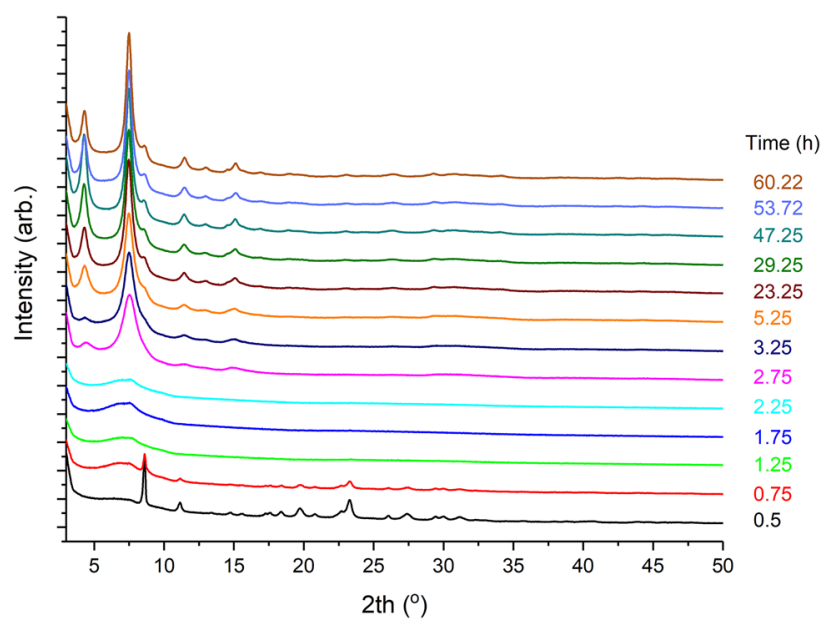

Figure 5. XRD patterns for the time-resolved samples removed from the STA-16(Ni) synthesis.

addition of the Ni salt. This reaction mixture became a lighter green overtime, again supported by the collected data. In these data a plateau is also observed suggesting no further change occurs after the first $5 \mathrm{~h}$. Combining $\mathrm{pH}$ and turbidity data suggest that the reaction has reached completion between 4 and $5 \mathrm{~h}$, not $72 \mathrm{~h}$ as previously reported-an improvement of $93 \%$. To determine if the reaction truly reached completion after $5 \mathrm{~h}$, a reaction was conducted in which samples were removed at various times. Figure 5 shows the PXRD patterns obtained from this experiment. It can be seen how peaks indexable to the STA-16 linker can be observed up to $1.25 \mathrm{~h}$. From 0.75 to $2.25 \mathrm{~h}$ a broad Bragg diffraction peak is observed at $\sim 7^{\circ}$ th, with significant diffraction from the STA16 framework only being observed from $2.75 \mathrm{~h}$ onwards. At $5 \mathrm{~h}$ a well-defined STA-16 framework is observed with increasing reaction time not producing further diffraction information. These data also allow for further elucidation of the crystallization process for these materials. It can be seen how amorphous material is observed prior to $2.25 \mathrm{~h}$ with a peak indexable to STA-16 (Ni) observable after $2.75 \mathrm{~h}$. It is within this time window that 


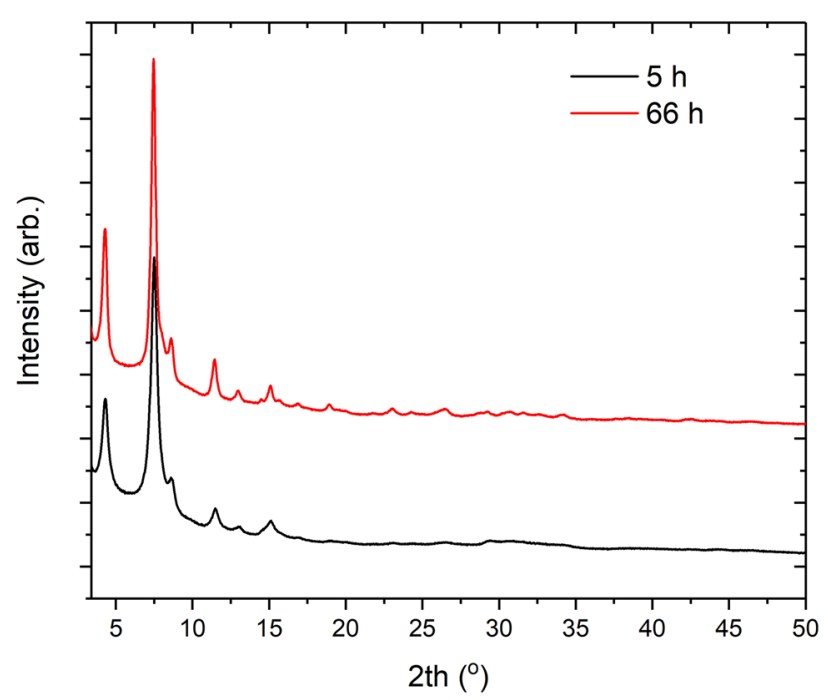

Figure 6. PXRD patterns for Entry 3 produced at 5 and $66 \mathrm{~h}$.

a drop-in turbidity is observed which is consistent with a critical concentration of metal and linker allowing for the formation of crystalline MOF.

Two small-scale reactions were conducted to determine if material produced at $5 \mathrm{~h}$ was comparable to material produced at longer reaction times. The reagent quantities used for these experiments are specified in Table 1 Entry 3. PXRD patterns can be seen in Fig. 6 which show that crystalline material is produced at both short and long reaction times. Importantly the yield of these materials remains constant with the $5 \mathrm{~h}$ and $66 \mathrm{~h}$ reactions producing yields of $27.08 \%$ and $30.31 \%$ respectively. Reaction times above $5 \mathrm{~h}$ must therefore result in little to no further material production. The increased reaction time does result in sharper peaks observed in the XRD patterns suggesting a growth in crystallite size with time. The crystallite size was determined after Rietveld refinement of the powder patterns using an integral breadth approach, Supplementary Fig. S4 online. Samples produced at $5 \mathrm{~h}$ and $66 \mathrm{~h}$ have calculated crystallite sizes of $9.854(69) \mathrm{nm}$ and 12.226(87) $\mathrm{nm}$ respectively. The increases in diffraction data at higher 2 th values indicate more long-range order is present in material produced at longer reaction times. Both the sharper diffraction peaks and the production of little to no extra material being produced is consistent and predicted from the observed plateau in the turbidity data.

Physisorption data, Fig. 7, collected on both samples show a difference of just $2 \mathrm{mmol} / \mathrm{g}$ at $\mathrm{p} / \mathrm{p}^{\circ}=0.1$. This increase in uptake may be due to improved long-range order; however, it should not be viewed in isolation. Ultimately these data show that a 13 fold increase in reaction time only produces a $16 \%$ improvement in $\mathrm{N}_{2}$ uptake. This shows how material produced after $5 \mathrm{~h}$ possesses almost all the characteristics needed from this material with only diminishing returns as reaction time increases.

To demonstrate the applicability of this approach in monitoring other MOF preparation routes a zeolitic imidazole framework (ZIF) was prepared and the reaction monitored using the apparatus. ZIFs, a subclass of MOFs, are typified by their zeolite-like structure and have been shown as interesting candidates for a range of application including gas separation and storage ${ }^{27}$. ZIF-8 has been widely studied and consists of tetrahedral $\mathrm{Zn}^{2+}$ ions connected by 2 -methylimidazole linkers in a porous sodalite topology ${ }^{28}$. Supplementary Fig. S5 online shows turbidity, temperature and $\mathrm{pH}$ monitored over the course of a synthesis of ZIF-8. Supplementary Fig. S6 online shows the XRD and Rietveld refinement collected from the material produced during this reaction. It can be seen how a phase pure ZIF- 8 material was produced during the reaction.

These data demonstrate changes in turbidity and $\mathrm{pH}$ that continue over the 200 -min reaction time while the temperature remains constant. These data demonstrate the applicability of the method to other MOF systems, not just STA-16(Ni).

These results show that, after only one experiment with our bespoke, budget data logger, the optimum reaction time for the preparation route of STA-16(Ni) can be predicted. This allowed for the reaction time to be decreased from 72 to $5 \mathrm{~h}$ with minimal impact on the quality of the final product—an improvement of $93 \%$. To reach this conclusion previously would have required the removal of sample at various times and collecting and analyzing ex situ XRD patterns. This shows how the in situ monitoring apparatus described in this paper can result in time saving for a very small upfront cost as well as reducing the resources required to reach these conclusions. This key result demonstrates the value of budget in situ reaction monitoring allowing valuable insight to be gained at minimal expense.

\section{Conclusions}

In conclusion we have demonstrated the ability to improve the MOF synthesis route of STA-16 by using budget in situ reaction monitoring. By using a range of sensors, we maximized the chances of observing a reaction critical process that can lead to direct optimization after just one experiment. This is a significant time saving that, in both industry and academia, will result in a sizable advantage within an agile and fast paced research 


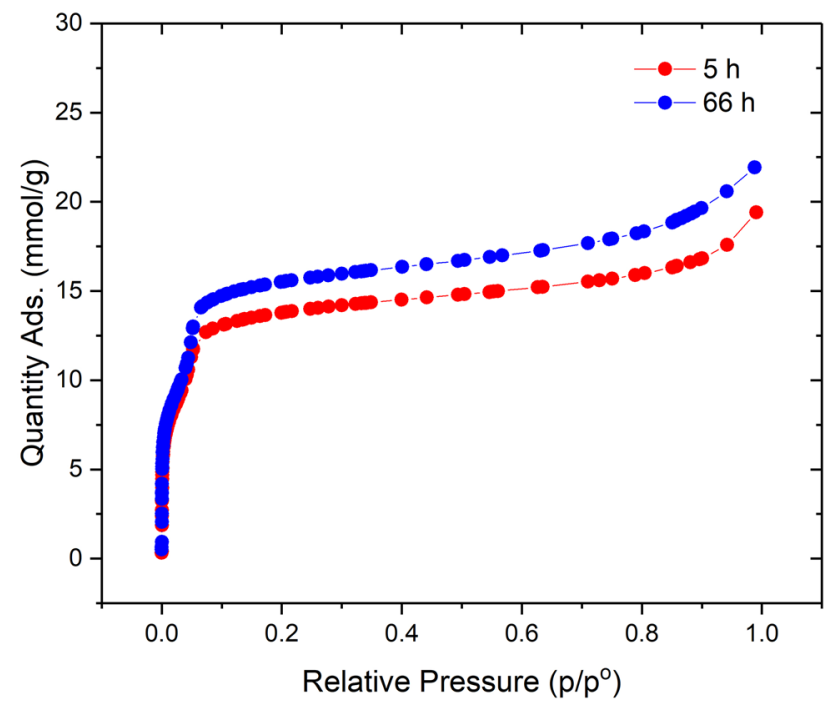

Figure 7. $\mathrm{N}_{2}$ isotherms collected on Entry 3 produced at 5 and $66 \mathrm{~h}$.

environment. This methodology is applicable to a wide range of other MOF systems as well as other systems where a precipitation reaction occurs, such as the production of metal oxides, zeolites and polymerization reactions. We have demonstrated this by monitoring the production of the MOF ZIF-8. Ultimately, by reaching the conclusions demonstrated above, less time, energy and resources are used to advance chemical science and bring possible products to market.

The reported apparatus is not without its limitations. While calibration is conducted prior to these tests, bespoke instruments lag behind their commercial counterparts which achieve better statistical validation of calibrations ${ }^{29}$. Valuable insight was gained into the STA-16 system and such insight is clearly of benefit to industry scientists working to improve synthetic conditions as well as scientists in academia working at the bench.

Further improvement to this apparatus could include onboard logging via a SD card, reducing the need for a connected computer and Internet of Things modifications could allow this apparatus to be monitored remotely. Additionally, the Arduino ${ }^{\circledR}$ offers many outputs, not just inputs. It is trivial to connect and code pumps or other devices to, for example, add reagents when certain conditions are met, enable samples to be extracted automatically or alter temperature and stirring rates depending on measured experimental variables.

All these processes are currently available in commercial offerings however the ability to quickly prototype and test equipment at the bench can be valuable to research scientists across multiple disciplines and institutions.

Received: 11 December 2019; Accepted: 3 September 2020

Published online: 15 October 2020

\section{References}

1. Kubínová, Š \& Šlégr, J. ChemDuino: adapting arduino for low-cost chemical measurements in lecture and laboratory. J. Chem. Educ. 92, 1751-1753. https://doi.org/10.1021/ed5008102 (2015).

2. Kang, S.-J., Yeo, H.-W. \& Yoon, J. Applying chemistry knowledge to code, construct, and demonstrate an arduino-carbon dioxide fountain. J. Chem. Educ. 96, 313-316. https://doi.org/10.1021/acs.jchemed.8b00663 (2019).

3. Costa, S. C. \& Fernandes, J. C. B. Listening to pH. J. Chem. Educ. 96, 372-376. https://doi.org/10.1021/acs.jchemed.8b00641 (2019).

4. Soong, R. et al. Combining the maker movement with accessibility needs in an undergraduate laboratory: a cost-effective text-tospeech multipurpose, Universal Chemistry Sensor Hub (MUCSH) for students with disabilities. J. Chem. Educ. 95, $2268-2272$. https://doi.org/10.1021/acs.jchemed.8b00638 (2018).

5. Salvador, C. et al. Open ISEmeter: an open hardware high-impedance interface for potentiometric detection. Rev. Sci. Instrum. 87, 055111. https://doi.org/10.1063/1.4952419 (2016).

6. Meloni, G. N. 3D printed and microcontrolled: the one hundred dollars scanning electrochemical microscope. Anal. Chem. 89, 8643-8649. https://doi.org/10.1021/acs.analchem.7b01764 (2017).

7. Rosa, T. R., Betim, F. S. \& Ferreira, R. D. Q. Development and application of a labmade apparatus using open-source "arduino" hardware for the electrochemical pretreatment of boron-doped diamond electrodes. Electrochim. Acta 231, 185-189. https://doi. org/10.1016/j.electacta.2017.01.180 (2017).

8. Mercer, C. \& Leech, D. Cost-effective wireless microcontroller for internet connectivity of open-source chemical devices. J. Chem. Educ. 95, 1221-1225. https://doi.org/10.1021/acs.jchemed.8b00200 (2018).

9. Johnson, T. et al. Improvements to the production of ZIF-94; a case study in MOF scale-up. Green Chem. 21, 5665-5670. https:// doi.org/10.1039/C9GC00783K (2019).

10. Li, H. et al. Recent advances in gas storage and separation using metal-organic frameworks. Mater. Today 21, 108-121. https:// doi.org/10.1016/j.mattod.2017.07.006 (2018).

11. Farrusseng, D., Aguado, S. \& Pinel, C. Metal-organic frameworks: opportunities for catalysis. Angew. Chem. Int. Ed. 48, $7502-7513$. https://doi.org/10.1002/anie.200806063 (2009).

12. Wu, M.-X. \& Yang, Y.-W. Metal-organic framework (MOF)-based drug/cargo delivery and cancer therapy. Adv. Mater. 29, 1606134. https://doi.org/10.1002/adma.201606134 (2017). 
13. Cattaneo, D. et al. Tuning the nitric oxide release from CPO-27 MOFs. RSC Adv. 6, 14059-14067. https://doi.org/10.1039/c5ra2 4023a (2016).

14. Bai, Y. et al. Zr-based metal-organic frameworks: design, synthesis, structure, and applications. Chem. Soc. Rev 45, $2327-2367$. https://doi.org/10.1039/C5CS00837A (2016).

15. Han, Y., Li, J.-R., Xie, Y. \& Guo, G. Substitution reactions in metal-organic frameworks and metal-organic polyhedra. Chem. Soc. Rev 43, 5952-5981. https://doi.org/10.1039/C4CS00033A (2014).

16. Van Vleet, M. J., Weng, T., Li, X. \& Schmidt, J. R. In situ, time-resolved, and mechanistic studies of metal-organic framework nucleation and growth. Chem. Rev. 118, 3681-3721. https://doi.org/10.1021/acs.chemrev.7b00582 (2018).

17. Yeung, H. H. M. et al. Control of metal-organic framework crystallization by metastable intermediate pre-equilibrium species. Angew. Chem. Int. Ed. 58, 566-571. https://doi.org/10.1002/anie.201810039 (2018).

18. Wharmby, M. T. et al. Synthesis and crystal chemistry of the STA-12 family of metal $N, N^{\prime}$-piperazinebis(methylenephosphonate) $s$ and applications of STA-12(Ni) in the separation of gases. Microporous Mesoporous Mater. 157, 3-17. https://doi.org/10.1016/j. micromeso.2011.12.003 (2012)

19. Groves, J. A. et al. The first route to large pore metal phosphonates. Chem. Commum. https://doi.org/10.1039/B605400E (2006).

20. Wharmby, M. T., Mowat, J. P. S., Thompson, S. P. \& Wright, P. A. Extending the pore size of crystalline metal phosphonates toward the mesoporous regime by isoreticular synthesis. J. Am. Chem. Soc. 133, 1266-1269. https://doi.org/10.1021/ja1097995 (2011).

21. Miller, S. R. et al. Structural transformations and adsorption of fuel-related gases of a structurally responsive nickel phosphonate metal-organic framework, Ni-STA-12. J. Am. Chem. Soc. 130, 15967-15981. https://doi.org/10.1021/ja804936z (2008).

22. Beier, M. J. et al. Aerobic epoxidation of olefins catalyzed by the cobalt-based metal-organic framework STA-12(Co). Chem. Eur. J. 18, 887-898. https://doi.org/10.1002/chem.201101223 (2012).

23. García, E. J. et al. Role of structure and chemistry in controlling separations of $\mathrm{CO}_{2} / \mathrm{CH}_{4}$ and $\mathrm{CO}_{2} / \mathrm{CH}_{4} / \mathrm{CO}$ mixtures over honeycomb MOFs with coordinatively unsaturated metal sites. J. Phys. Chem. C 116, 26636-26648. https://doi.org/10.1021/jp309526k (2012).

24. Etxeberria-Benavides, M. et al. PBI mixed matrix hollow fiber membrane: influence of ZIF-8 filler over $\mathrm{H}_{2} / \mathrm{CO}_{2}$ separation performance at high temperature and pressure. Sep. Purif. Technol. 237, 116347. https://doi.org/10.1016/j.seppur.2019.116347 (2020).

25. Coelho, A. TOPAS and TOPAS-Academic: an optimization program integrating computer algebra and crystallographic objects written in C++. J. Appl. Crystallogr. 51, 210-218. https://doi.org/10.1107/S1600576718000183 (2018).

26. Cheary, R. W. \& Coelho, A. A fundamental parameters approach to X-ray line-profile fitting. J. Appl. Crystallogr. 25, 109-121. https://doi.org/10.1107/S0021889891010804 (1992).

27. Kwon, H. T. \& Jeong, H.-K. In situ synthesis of thin zeolitic-imidazolate framework ZIF-8 membranes exhibiting exceptionally high propylene/propane separation. J. Am. Chem. Soc. 135, 10763-10768. https://doi.org/10.1021/ja403849c (2013).

28. Park, K. S. et al. Exceptional chemical and thermal stability of zeolitic imidazolate frameworks. Proc. Natl. Acad. Sci. U S A 103, 10186-10191. https://doi.org/10.1073/pnas.0602439103 (2006).

29. Pearce, J. M. Building research equipment with free, open-source hardware. Science 337, 1303-1304. https://doi.org/10.1126/scien ce.1228183 (2012).

\section{Acknowledgements}

The authors are grateful to the JMTC analytical team for various data collected. T.J. would like to thank Huw Marchbank for fruitful discussions related to XRD data visualization.

\section{Author contributions}

S.P., H.Y., S.B. and T.J. designed the project. F.M.M. conducted the research. F.M.M and T.J. contributed equally to writing the paper. All co-authors edited the paper.

\section{Competing interests}

F.M.M, S.B, S.P and T.J. work for a company with an interest in the commercialization of MOF materials. H.Y. has no competing interests to declare.

\section{Additional information}

Supplementary information is available for this paper at https://doi.org/10.1038/s41598-020-73780-z.

Correspondence and requests for materials should be addressed to T.J.

Reprints and permissions information is available at www.nature.com/reprints.

Publisher's note Springer Nature remains neutral with regard to jurisdictional claims in published maps and institutional affiliations.

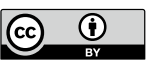

Open Access This article is licensed under a Creative Commons Attribution 4.0 International License, which permits use, sharing, adaptation, distribution and reproduction in any medium or format, as long as you give appropriate credit to the original author(s) and the source, provide a link to the Creative Commons licence, and indicate if changes were made. The images or other third party material in this article are included in the article's Creative Commons licence, unless indicated otherwise in a credit line to the material. If material is not included in the article's Creative Commons licence and your intended use is not permitted by statutory regulation or exceeds the permitted use, you will need to obtain permission directly from the copyright holder. To view a copy of this licence, visit http://creativecommons.org/licenses/by/4.0/.

(C) The Author(s) 2020 